\title{
Aspectos del ejercicio obstétrico en clientela civil
}

\author{
Por los doctores RAFAEL RAMIREZ MERCHAN \\ y SERGIO LAMUS GUERRERO
}

La atención de los partos en la elientela particular, en clinicas especialmente dotadas, difiere. dende luégo, de la atención hospitalaria. por rayones obvias, que no es necesario enumerar. Ios resultados finales tanto maternos comto fetales han de sere por ronsiguiente. más halageïnon que los obtenidos en la -lientelat hospitalaria y lon índices res. pectivos deben reveiar adelanto de la téeniea y la precisión y efieieneja de la especialidad.

Es claro que en el ejercicio particular. sube la incidencia do las intervenciones. al propio tiempo que disminuye la de las romplieaciones y que bajan los in. diees de morbosidad y de mortalidad. Iresentarlos esenetamente estos indioes.

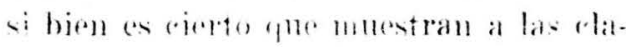
ras ol éxito final. podrían alamar. on e) sentido de que ran siendo cadia dia nás escasos los parter espontáneos y que estamos contribuyendo romo espercialistas a contrariar las leyes naturalos, entorpeciendo la fisiología. Sin cm. hargo, esta alarma no se justifiea. ?orque si nos aturiéramos a que of parto debe emuplirse simupe de una man"ra fisiológica. como sc emmplen las demás funciones, hatria que considtear romo ronsecuencias d. !n fisiología los desgarros perintales que, ocurren asi en ol (iento por ciento de las primíparain que paren sin aỵda racional y cioutifica: liabría que aceptar también cono consereneneias de la fisiología. las retencio nes de placenta parcial o total que, se producen en las paciertes que soportan sin asistencia idónea, su alumbraniento: tendria que ser también fisiológiea la muerte del producto por circulares del cordón. si no se diagnostica opurtunamente el sufrimiento fetal y si no se recurre al arte para corregir estas paradojas de la naturaleza. En fin, tendríamos que aceptar como conseruencia de lo fisiológico, la imposibilidad del parto en las presentaciones viciosas y "W las deformaciones pélviøas y, la ronsiguiente muerte de la parturienta y de stl hi.jo como resultado de un caprieho do lat naturaleza. en mata funcion roua rollerión debería ser tan corrertal eomo la de las demás.

No es el parto una función comparalide a todas las demás, porque ninguna Heva en sí tántos peligros ni tan grave amenaza de muerte, así romo timpoeo ninguma otra es susceptible de tan amplia perturbación. No se puede esperar a) parto, para no mencionar los riesgros de la gestarión, ron la impasibilidad (•on que se espera ma miecoín espomtínea o con la indiferencia con que se 
pera de los endriduos normales. quer -iga sll rurso la sistole y diástole carcilia a y los domás movimimentos immanomtes de la vida.

lat grestar.ión, ol parto y el puerperin repuierent rontrol, vigilancid, rondurerión. correverom do ammolías amm en las mujeres remsideradas por torle asperto nommales. y de romsigniente. se justifiea la esporalizarion para impediir los desastres, sin abusar tampoos) de lon medios rentífieos. No se justifira (n rambio el esperialista a toclo tran(.xpectante y que espera la eompliracion para intervenir; su misión funGamental estai en prevenir las complivaciome y vale más que intervenga alglunas veres sin apremiante neresidarl. a flle lo haga viando ya han ormerido trastolnos irreparables.

El relativamente alto porcentaje dit intervoneiones obstétricas que, sobre lon rasos ransideraremos en este trabajo, así eouno la activa conducerión de los partos i-pontáneos, descubren las modalidade del ejercicio en clientela particular .n beneficio de la madre y del Jijo y. Ilaman poderosamente la ateneión sohte la comveniencia de mejorar los servios hospitalarios hasta prestar allí $u n$-nidalo similar al que se presta on elinelela particular y en ampliarlos hasta tal punto que se araben los sorvi.io domiciliarios y la intervención de eomulronas que tanto mal callsall.

Sobre riento moventa y ocho (198) casos obstétricos, total de las historias rlínirar te Ramírez Merchán, en la Clíniva d. I a Magdalena, año de 1950, vamos a entudiar su distriburión, las conductas seguidas. los indices y los resultados obtenidos, aomentándolos brevemente an $u n$ sentido orientado hacia Ja printira rlíniea de la especiabidad.
$11,1211,1,12,2,20.120$

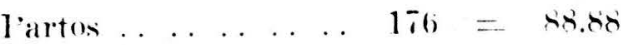

Abortos . . . . . . . . 18 : 9.04

Amenaza de aborto . . 3 : 1.52

Amenaza de parto pre-

maturo . . . . . .

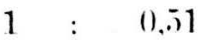

Según la torminacón del mbatrazo minolltinuos:

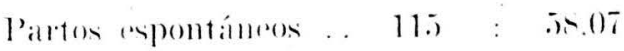

Embarazo gemelar .. 4 : 2.19

Forreps. . . . . . th : 2t.2t

C'esáruas .. . . . . . . 8 : 8 : 4.04

versiones internas .. 1 : 10.51

Abortos .. . . . . . . $18 \quad$ : 9.09

Amenaza de aborto . . $\quad 3 \quad$ : $\quad 1.52$

Amenaza de parto pre-

maturo ........

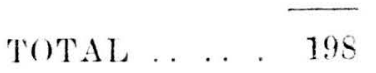

0.51

\section{CAPITTIO I}

PARTO D:LONTANEO

1,a noción actual del parto ispontámeo no es exactamente la rlánica, por rnanto hoy en día se dispone die algen-

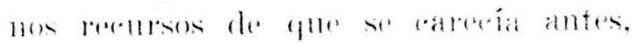

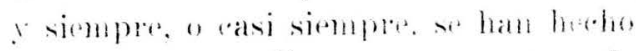
indiraremes medicamentoses anters de Ja ferminareion del parto a más de que. on la gran mayoría, la expulsión th sil jeriodo final se have bajo anestesia y" la episiotomía, profiláctica de los deszarros perineales. en partos hasta allí 110 intervenidos, restringo más aún el antigun eoncepto del parto espontineos.

En la rutina de las elinicas. a toda praciente que inicia trabajo de parto so l. apliea ma gran lavativa de aglla jabonosa $\because$ si el trabajo se prolonga por

\section{$\overline{124}$ Obstetricia y Ginecología}


más de 12 horas. esta indicación se repite. a fin de que las vías intestwales lajas estén libres - para el tirmpo del desprendimiento. La costumbre de apliar lavados repetidos hasta obtemer ma limpleza de mayoles garantias, no as aleptacla por mosesta elientela rivil

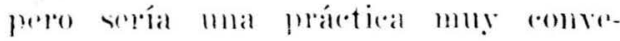

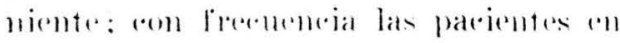

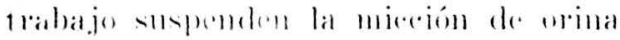

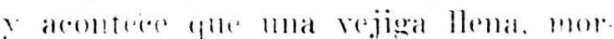
tificada intermitentemente por las enent raceiomes nterinals, a más de sere 111 obstáculo pala la progresión del polo que se presenta, almenta naturalmente el sufrimiento de la parturienta $y$ predispone a las paresias vesicales del puerperio con la comsiguiente retención: por estas razones merece particular atención este detalle y si la paciente no puede arinar espontáneamente es preciso pasaile la sonda.

Aun collodo parezea supertluo, es romvenimente anotar (juc la parturienta lebe guardar ma dieta alimenticia cer(•xna al aymo: 10 es bien que ingiera rosa distinta de líquidos, salvo en los arsos en que se puede prever que el trabajo se prolonga por wás de 12 horas. Es de elemental compremsión que la aretividad fúsiea y psíquiea de la patrionte. eonoentrada en el trabajo del jarto. no permite $m$ buen funcionamiento digestivo y el resargo immeresirio suma a los padecimientos del parto los de la indigestión y. si se tiene en miras la anestesia para el desprendimiento. un estómago lleno compliva imemarrablemente las posibilidades de la anestesia; la muerte por obstruceión de las vías respiratorias, eon las sustancias romitadas, no es ma lejana posibilirlan. Cuando la prolongaraión del trabajo ha"e posible la deshidratación de la par- furienta se debe prevenm aquella mediante la venoclisis de soluciones de dextrosa ; esto se have impositivo cuando el trabajo evoluciona ron la bolsa de las acuas rota prematuramente.

Tarefes ragimales. Es costumbre prarticar el tacto varinal deste la iniciacoón (iel trabajo. a intervales raciomales a mpustos por la reblucion del mismo. El cammen inieial permite eomprobar la efiedendia de las eontracoiomes. determinar la normalidad del canal. apre•iar con aproximación la forma del trabajo en lo que respecta a las caracteristicas del euello. valeular la duración de la dilatación. (omprobar la clase de presentación. la integridad o ruptura de las membranas y descartar las posibilidades de moreorón procidente a prolabado. En las primigestantes o en las recomocidas estrerehas, por el tacto combinado se determinam las relaciones pel. vifetales y simplemente por tacto. la posibilidad de eneontrar 110 promontorio alcanzable.

Se han denigrato los tactos vaginales y hay quiemes practioan el taleto ree1al: respetando a quiemes así pooerelon. mo los imitames ni los imitaremos. porque, en prinuer lagar. las pacientes los

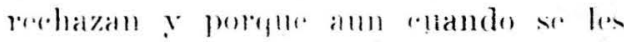

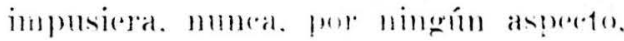
smomintra todos los latos que propor-

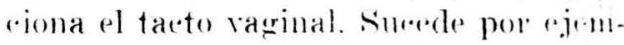
plo, qu" si no se diagruostica oportmanmente un cordón providente se ponr en peligro la vida del foto por miedo al

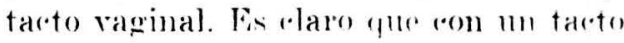
rectal no polliamos hacer un diagmóstieo previso d. esta modalidad. El de. rautado pelieron de infereoion por motiva ciel tacto vaginal. es a mestro sentir, Y por la experiencia diaria, un mifo. El tar.to se practica en óptimas condicios- 
mex de asepsia, previame ite se ha hecho el rasurado. se han hecho embrocaciones de la regrión eom una solución desinfectante y nua buena téniea impide yue los dedos que se introduren, rocen los bordes volvares y have que penetren directanente m la vagina, la emal, en an embarazo bien vigilado. es acida con flora defousiva tavorable. Alemas. la practica comriente toma mediclas profilateticas del puerperio en forma qui" veremos aclelante y que permite reducir asi a cero la morbosidad purperal.

Se permite que la paciente an trabajo, si no ha habido ruptura de la bolsa deambule libremente en su pieza durante el primer periodo, segúm la clasifjearión americana de tres periodos y, ain a el segmolo periodo en la primera parre. la pariente dobe mantener tal libertat.

fomliod del trabajo. En la Clíniea dr. lad Magelalend es rostumbre anotar pesólicamente. por iniciativa del Direc. tor. en uri cmalro que se lleva en la sala de partos y yue facilita la elaboravion letallada de la historia clínioa, la hora da la indoiateion, las caracterítiras de las contratedones en enanio a imensidad. ritmo ! duración. la evoluriom

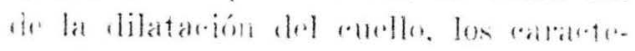

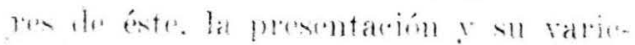

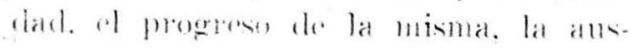

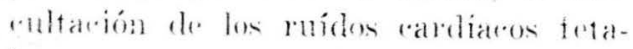
les eon sus ealacteristiras y las pres-

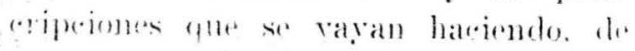
matuera que de $101 \mathrm{~s}$ solo golpe de vista. a) obstetra se da cuenta de la mareha ref trabajo en todos sus detalles.

Medicacion. Para reducirnos al eomentario de las historias elimiras ylle nos han serride de tema..el elladro siguiente muestra la forma de medina‘ión : $\begin{array}{lllll}\text { Trabajos inducidos } & \ldots & \ldots & 6 & 5.22\end{array}$

l'artos terminados sin me-

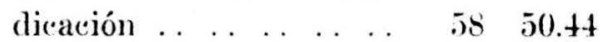

lso de ocitócicos . . . . . . $2424 \quad 20.86$

I'so de sedantes .. . . . . 17 14.78

Medicación mixta (sedan-

tes-ocitócicos) $\ldots \begin{array}{lllll}\ldots & \ldots & 10 & 8.70\end{array}$

TrT.।.

115100.00

La incucción del trabajo se ha practicado en pacientes que han llegado a término o Jo han pasado, en las cuales existe ma presentación de vértice ent. "ajada o siquirera insinuada $y$, un cuetho borrado y blando. El resultado ha sirto de éxito en el ciento por ciento de los casos; es rierto que éstos son mur pocos. pero el procedimiento ha sido ampliamente extudiato en la tesis de grado del doctor Manuel dosé Gómey Palacino, presidida y dirierida por mon de nosotros. La indueción se ha hecho con la administración por vía endove. nosa en goteo lento, de una solución de dextrosa al it/ o al $10 \%$, fraseo de 500 c. c.. al rual se arlicionam 3 mnidades de Pitoén; no insistimos sobre detalles de téenica expmestos en la reitadia teris.

Los parter terminados sin medieacion

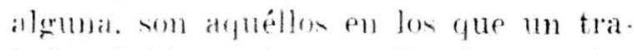
hajo ripjdo y sin romplicariones, soldmente exige, all la rondueción moderna del parto. la anestesia para el período del desprendimiento. Conviene si, anotar que de los partos aspontáneos. segúm el sentide de la palabra expiendio antes, tan sole 10 50.4t\% terminaron sill mertivareicin.

Ocitrcicos. Lal cautela y rigor que deben mediar para ol uso de los oeitio ricos se pueden apreciar ampliansente, 
ru el estudio de estas historias rínicas. En primer lugar. la indicación de nu oritócico requiere muy buen sentido $\cdot$ lí. nico y experiencia en la especialidad: ell segundo lugar. sul dosificación ha dr. ser muy prodente y la respmesta de carla paciente en partionlar. es la que de. romina sule repetioión a la preseinder. fia. ('omo norma general, no deben nare. se en el primer periolo. simo en rases comprobados de inerelia primitiva. y entonces prácticamente se signe la ?anta de la inducción del trabajo. En tales ‘asos, hemos prescindido. casi por comepleto de su empleo a peyueñas dosis subcutáneas. porque se corre el riesur. de perturbar la dinámica nterina y pro. verar el sufrimiento fetal. La sieniente. historia da ma idea de la forma de con. sureir la indurejón:

Caso número 67. Historia número 610. J'rimigestante a término. Feto vivo. Llega a la clínica el 18 de mayo de 1950, en la tarde. Se hizo tacto varinal que reveló el estado de comienzo de la Gilatación. Durante la noxhe hubo wontracojones muy expaciadas. Durante u lía 19. dolores esporádicos a hipotomio ir la matriz. En la noche clumua libr. mente. A las 9 a. M. del día 20. latioti va jabonosa. A las 10 y 15 a. m. inducrioin com dextrosa al $10 \%, 500 \quad \cdots \quad \cdots$.. más 2 unidades de pitocin. A las 14 " uspendió el goteo; alcanzaron a parat. :300 a. c. Desde la iniciación de la velioclisis se regularizó el trabajo (on bur. lias contrarciones rada 2-3 minutos: pur - wto. se suspende y se aplicó nua ampolla de Demerol a las 14 y 30. A la 18 y 30, nuevo tacto vaginal: mismo stado anterior, cuello blando y delga. do, 3 centímetros de dilatación. A las
2:) horas se orelena linal ampollat die Mis Y ma cápsula de nembutal. para dar reposo a la pariente yue se siente agetada. Ia expulsióm no se ha inieiado. pues la dilatación permanece lo mismo. El sosiego fuo muy estaso. Mayo 21, a las 3 a. m.: se le apliea mata amplata

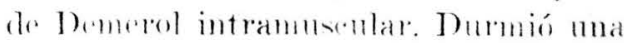
homa y media. A las 7 y :3.). so para a la sala y se hace tarete vaginal que re vela: dilatación enmpleta. bolsa intewra. Por anestesia ron inhalaciones intermitentes, se esperó el descenso y rotarión y. ya en (). P.. se aplicó fóreeps de desprendimiento: se extrajo niña de 4.320 gramos que respiró espontáneamente. Anestesia: (Cielopropano-02.

Jos ocitócicos empleablos y, nos referimos al uso del Pitorín. en dosis de nua a dos miclades. se han aplicado en la sala de partos eom la pareiente en la mes operatoria y al iniciar la analgesia o la anestesia. En tal forma, equilibrada la areión soclante del anestésico y la excitante del ocitócico. se subsana la acejom del primero y se asegura ol ritmo normal de las eomtracejones. a más de que se asegne a rápielo desprendimiento de la plarenta. C'nando

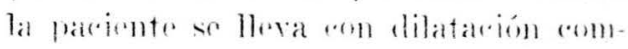
plota y bolsa do las agmas interga. se romperesta, se apliean nua o los moidades do pitooin y se inieia la amaleresia; rllando ésta se halle ron gaves. la parjente se ayuda si se have an forma intermitente. Cuando usamos el pentothal, en la forma romo lo commontaremos aparte, signen actuando las eomtraceiomes uterinas y la pareiente. libre de aprehension ! de dolor hare enfuer-

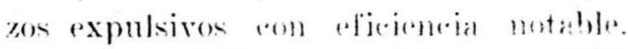
Ia transeripción de dos historias clini. ras nos permite remoer mejor esta manera de actuar. 


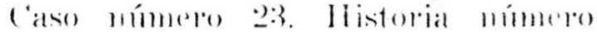
148. Muligestante a término, con ferto vivo. Para IV. Febreme 5 de 1950. Ho.

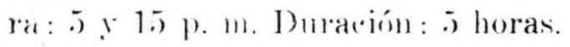

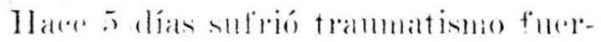

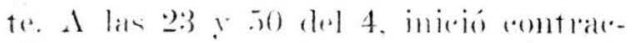

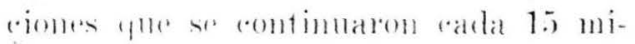

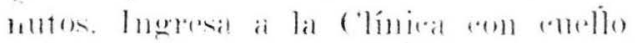
en 6 e entinnetros de dilatarion. peresentareion ()lla. bulsa integral y dolomes rada jommos. Sir pasa a la sala, a las 4 y 45 ; rmptura expontínea de la bolsa. dilatación completa. Se aplican 2 midades de pitoeín y bajo anestesia se verifica el desprendimiento en O. P., de $u m$ niño de 2.340 gramos que respira expontaneamente. Alumbramiento espontín.o a los 4 minutos.

Amestesia: (ciclopropano-0.2. 1)nareión 10 minntos.

Caso numero 172. Historia número 1.f(1). Multigestante a término. Feto vivo. l'ara III.

Se hace tacto vaginal ol 26 de noviembre. a las 23 y 15: anello biando, bo-

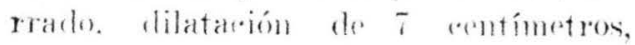
memblatuas intergas. presentarion en(ajadi, a ()]I)!?

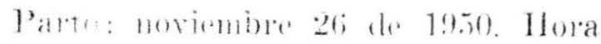
2:3 y tis. Domateion: 12. homas.

Sir farad a la sala ron dilatarión rasi fompleta. membranas integrats, plesentia ión insimala. Se rompen artifieialmente las membranas, se completa la dilatarion y se aplican 2 midades de

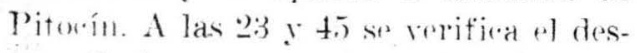
prendiniento espontánes de nua niña

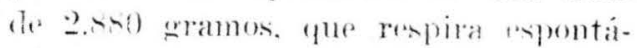
leamente. Alumbramiento epontáneo a los 1.5 minutos.

Anestesia: Pentothal-02. Muration: 15 minntos. sidentes. las sedantes y espasmolititieos se han empleate rasi sistemátieamente en las pareientes irritables, también (mande quiera que las rontranedomes uterinas tienen temelemeia a la hifuersistolia primitiva o seemelaria 0 "mande son demastatio enérerieas. (0) foligro de mion-lonia, fllando hay espate-

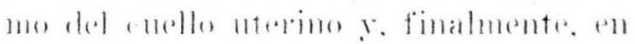

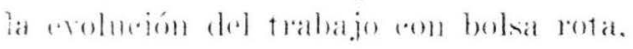

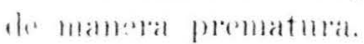

Las pacientes irritables. especialneente las primerizas, suelen iniciar trabajo en forma tumultuosa e inefectiva, (*on muestras de gran desesperación; en tales casos, se usa un barbitúrico de tipo amital, nembutal o seronal a las dosis te 10 a 20 eentigranos por vía oral ieste el principio del trabajo. asociacio " mna ampolla de demerol por vía in-

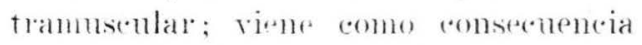
nu periodo de ahna ye de reposo que (la comfianza a la paciente, la cual suel. reanudar el trabajo con contraceiones espaciadas y muy tolerables; si ulteriormente reineide la irritabilidad, la administración medicamentosa depenio del grado de evolución en que vara a) trabajo. porque si el enello ha borra(io totalmente y la lilatación ha pasado

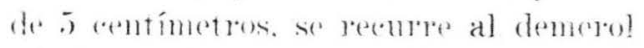
melminintrado por vía endorenosa it la dosis d: 10 miligramos o sea la ampolla de 2 * * dismeltos en 8 r. ध de agua destilarla $y$ aplicedos rápidamente los 5 prineros centímetros y. muy lentamente el resto, ron lo enal se controla la reaceión a la droga y se evita la produreión de vómito, muy frecuente si se aplixa an forma rápida. El resultado es frabeamente alentator: la calma se produre inmediatamente; las contrace romes continúan con ritmo normal y el (riello se dilata con pasmosa rapidez. 
en el más alto porcentaje we los casos. El demerol aplicado en esta forma es el mejor de los antiespasmódicos $\mathrm{y}$, acelera el parto siempre, con más o menos efectividad hasta tal punto que en ocasiones se resuelve en el curso de una a dos horas, después de su aplicación. Estat a veración es tan rierta que poetemos lar un promedio de durarión del trabajo del parto, en los espontáneos. interior a las estadísticas que conocenum. Tal promedio, como se vera en los ruadros adjuntos, es el de 8 horas 5 minutos, en un total de 23 primiparas $\because 93$ multíparas.

Si el trabajo no está avanzado, entonces so repite la administración del demerol por vía intramuscular, reserrando la vía endovenosa para cuando se llenen las condiciones anotadas; el efecte de la droga sobre el producto de la con \%pción no se hace sentir si se sigue tal norma. Empero. cuando ha habido repetición de la medicación o cuando se aplica estando muy cercano e] desprendimiento, debe desconfiarse de la anestesia por pentothal, porque la sima de acciones deprime el centro respiratorio fetal. En la tesis del doctor Eduardo Trujillo Erazo, sobre hiproamalersia, se estudia un procedimionto similar al que venimos anotando, con combinación del barbitúrico y del demerol. El doctor Trujillo apenas anota sti a..ión sedante, pero no pára mientes en la acción antiespasmódica efectiva sobre los espasmos cervicales y, sobre la evidente abreviación del tiempo de durarion del trabajo del parto.

Las historias siguientes transcritas en lo pertinente, muestran la acción del demerol por vía intramuscular.
Laso numero 14. nisloida nume 0 on. Primigestante a término. Feto vivo.

Parto: junio 2 de 1950. Hora: 16. Duración: 11 horas.

Llega a la clínica, a las 10 a. m., con buenas contracciones cada 15 minutos.

El tacto vaginal a esta hora reveló: ruello borrado, dilatación de 3 centímetros, bolsa integra, presentación de vértice insinuada. Para sedarla se ordena una ampolla de demerol intramuscular. Continía con buen trabajo. A esta hora (las 14), se hace nuevo tacto vaginal, que revela: cuello casi completamente dilatado, presentación insinuada, membranas íntegras. Se ordena otra ampolla de demerol. A las 15 y 45 se pasa a la sala con dilatación completa y pre. sentación descendiendo, bolsa rota. Se dan inhalaciones de Ciclo-02 intermitentemente durante 10 minutos mientras descendía más la presentación; una vez ésta en O. P., se hizo episiotomía bajo pentothal. Se produjo desprendimiento espontáneo de una niña de 3.240 gramos que respiró espontáneamente. Se aplica una ampolla de Pitocín subcutáneo. Después de esta inyección se produjo el alumbramiento espontáneo a los 8 minutos.

Anestesia: Pentotal-02. Ciclopropano02.

Caso número 175. Historia número 1.420. Multigestante a término. Feto vivo. Para IV.

Parto: noviembre 28 de 1950. Hora: 23 y 30. Duración: 23 horas.

Llega a la clínica a las 7 a. m. del 28 con trabajo irregular que se había iniciado a la 1 a. $\mathrm{m}$. del mismo día. A las 19, los dolores son cada 15 minutos. 
A las 20 horas y 30 se da una cápsuld de nembutal $y$ se aplica una ampolla de demerol intramuscular. A esta hora el tacto vaginal reveló dilatación de 4 centimetros, cuello borrado, bolsa ínregra, presentavión (OIDI' insinuada. A las 23 y 15 tacto vaginal: dilatacion eompleta, bolsa intarta. presentarión enrajala en (lll)l'. Se para a la sala y haju anestesia, despremelimiento expon-

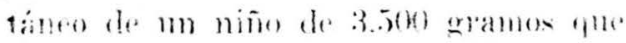
replira espontancamelite.

Alumbramiento espontánes a los is minutos.

Anestesia: Pentothal: 0.12 gramos. e.2. Iuración : 5 minutos.

Lita: ligero pstalo espasmódieo de las vas respiraturias de la paciente que, se eontrola mediante nua ampolla de morfina-atropina.

Hemos transerito de eleceión la historia anterior (case nimero 175) por su interés en el sentido de que contrariamos to dicho antes, del peligro de la anestesia por pentothal (vnando se inan repetido las dosis de demerol o conande se ha aplieado estando proximo el desprendimiento. Sin enbatron, en asto (*aw. no hubo efeete hatime sober of foto joorepla medio may corto epario antue

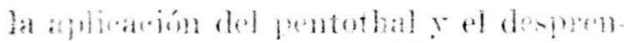
cimiento fetal, te manela que no alranzo a pasar a sll cireulation of presducto anestésieo. Hubo en cambio tendencia al espasmo de las vías respiratorias d. la madre. y es éste precisamente mo d. los incomvenientes anotados a la anestecia por vía endovenosa.

De la efinatia del demerol por la vía andovenosa hablan miny raro los rasos numeros 177 y 192 , que traseribimos en seguida.
Caso numero 17t. Historia numero 1.434. Multigestante a término. Feto vivo. Para 111.

Parto: diciembre 3 de 1950). Hora: $18 \mathrm{y} 10$

lelega a la clinica a las 11 a. m., ron dolores poro frevonestes. Presentación alta en ()ll C. Continna el trabijo inremolar deranter tomlo el diat hasta las ib

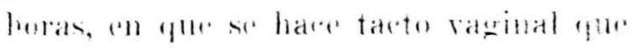
levela: dilatacion de 3 entimetros. enello grusso, incompletamente borrado y duro. Se le aplioa a las 17 horas. ma ampolla de atrinal. Los dolores se hacen más frecuentes. A las 17 y 40 se aplica una ampolla de demerol endovenoso. Continúan los dolores aumentaudo en frernencia e intensidad. Se hace nuevo tacto vaginal a las 17 y 55 : dilatación completa, presentación en el estreche inferior; bajo anestesid se rerifica al desprendiniento de $m$ niño de 3.260 gramos que respira espontáneamente.

Alumbramiento espontáneo a los 5 minutos.

Anestesia: Cielopropano-02. Dura(Fín: 10 minntos.

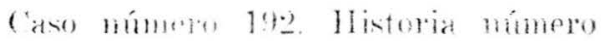
1.512. Sermoligestante a téruino. Frto vivo.

Parto: dicienber ez:3 de 1950. Horà: 12 y 10. Duratión: 2 holas 30 minutos.

Inició dolores a las 10 y :30 del 23 de

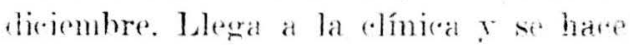
tacto vaginal: dilatarión de 6 etme. membranas rotas. $A$ las 11 y 1.5 s. apli"a ma ampolla de demerel ondowenoso. sigue el trabajo "n forma regular. La dilatarión es eompleta a las 12 y la pre critarión está enrajara. Bajo auestesia 
se prodice el parto espontáneo de una uiña de 3.060 gramos que respira espontáneamente. Se aplican 5 unidades de pitocín y 5 minutos después se produce el alumbramiento espontáneo. Pequeño deszarro de la horguilla.

Anestesia: Pentothal-02. Durarión: 30 mimmos.

Meneimanos of uso de sedantes romono Jos barbituricos y el demerol en la romduceión del parto con bolsa rota de manera prematura, y es esta una conducta digna de particular atención, por cuan10 no es rara la ocurrencia de la inercia paradójica, cuando se rompe prematuramento la bolsa de las aguas y, con una aparente calma de la paciente y en ausencia de contraceiones uterinas, hay wa evidente hipertonia que se exagera eon la menor excitación por ocitócjuos. La comsernenvia es la tetanización d. la matriz, el sufrimiento fetal y los puligros inherentes a la dinámica perturbada. Tales inconvenientes se obvian y los peligros se evitan, si en lugar de prefender estimular el trabajo con ocitóciecs, se procede lógicamente bajando el fono uterino con los sedantes y espasmolitiens, a fin de que el trabajo se inije to manera espontined.

Hedicarion mirta. En algumos vasos se ha mopleado ma medicacion mista de sedantes y ocitócions; la mayoría de las veces el ocitócico viene como mediación final en la sala dr partos, cuan fo no hay peligro de perturbar la dinamica utrina $y$ en casos muy raros los sodantes se han usado para moderar la aución muy enérgica del ocitócieo.. Jas historias numeros 111. 156 y 171, que van en seguida, muestran la manera como se ha usado la medieación mixta.
Casciónero 111. Historia número s.t. Seroundigestante a término. Feto vivo.

Parto: agrosto 13 de 19.50. Hora: $1 \mathrm{y}$ 10 a. m. Diración: 11 horas.

El 12 de agosto a las 10 y 30 ma ampolla de atrimal. A las 20, ma cápsula de menbutal. A las 23 y 30 m supositorio de trasentina.

El 1:2 a las 2 a. mi., se injuiarou los colores. La enterma pasa a la sala a las () y 45), del 13, con dolores cada 2 minutos, dilatación de 8 centímetros. Presentación encajada en OIIA. Membranas integras. Bajo anestesia se completa la dilatación, se rompen artificialmente las membranas $y$ se aplican 2 midades de Pitorín. $A$ la 1 y 10 a. m., se produce el ciesprendimiento espontáneo de un sino que respira espontánemente yo que peas 3.360 gramos.

Almubramiento espontáneo a los 5 minntos. Desgarro perineal de primer crado gua se sutura con catgnt en dos planos.

Anestesia: Ciclopropano-02.

Cact monero 156. Historia númere T.:30:3. Mnltigestantr a término. Feto vive. Para 111.

Parto: artubre 29 de 1950). Hora: $3 \mathbf{y}$ 5.5 a. m.

Se practiea tacto vaginal a las $11 \mathbf{y}$ 30) del 28 de octubre: dilatación de 4 centimetros, cuello grueso, presentación por encima de] estrecho superior en (MDP, bolsa intacta. Trabajo irregular. Nuevo tacto a las 2 y 30, del 29: dilatación de 6 centímetros, cuello grueso, duro, presentarión insinuala en (OJDP, bolsa intactá. Se ordena una ampolla 
de atrinal y una eápsula de nembutal. A los 20 minutos el trabajo se hace más frecuente e irregular; se pasa a la sala a las 3 y 30 con dilatación de 9 centímetros. bolsa intacta, presentación encajada. Se rompen artificialmente las niembranas y se aplican ¿ midades do Pitocín. Parto espontánece de un niño de 3.000 gramos que respira espontáneamente.

Ahimbramiento espontáneo a $\operatorname{los} 8$ minutos. Se aplica una ampolla de ergotrate y s midades de Pitoein intramuncular.

Anestesia: Cirlopropano-02

(as) mintero 171. Historiat numero 1 fort. Primigestante a término. feto vion.

Parto: noviembre 2.5 a 19.50. Hora: 14. Buratión: 14 horas

Inició trabajo el 25 a las 0 horas, en format lenta $y$ progresiva. Llega a la - linina a las 11 horas del 25 con trabaio womal y contraceiones cada ? a 4 minutos. El tacto vaginal a esta hora matrí allo biando, dielgato, dilatacom in ? centimetros membranas intremas presentarión enratiada on el estresten superior.

1 las 11 y 45 se le da una cápsila do minbutal $y$ a las 12 horas se aplica una ampolla de demerol endovenoso, obteniendose una sedación casi completa con commolencia. El trabajo sigue su crolución normal. A las 13 y 30 se inirian nuevamente contraceiones fuertes. A las 13 y 40 se pasa a la sala con: dilatacion completa, membranas integras, presentación en el estrecho inferior.
Se rompen artificialmente las membranas $y$ se aplican 2 unidades de pitocín. Se verifica el desprendimientu espontáneo de una niña de 3.100 gramos, que respira de manera inmediata. Alumbramiento espontáneo a los 5 minutos.

Se produjo un desgarro de la pared posterior de la mucosa raginal que se sutura con catgut simple número 1. El feto traía ma circular de] cordón en el cuello.

Alumbramiento. Tanto an los partos espontáneos como en los intervenidos con fórceps, se ha obtenido el alumbramiento espontáneo y rápido en un lapso de 5 a 10 minutos, como consecuen“ia del uso de 2 unidades de Pitocín, momentos antes del desprendimiento; A Pitocín que ayuda al desprendimien10 fetal, apresura también el desprendimiento y la expulsión de la placenta. En esta forma se evita el retardo del alumbramiento y se reducen al mínimo las complicaciones. Cuando. después de 10 o 15 minutos, no se ha logrado el alumbramiento ayudado con la expresión abdominal, se considera que hay atgo anormal; se revisa entonces el canal del parto; si hay una retracción del rmello, la anestesia profunda resuelve la complicación; mas si al ruello permanece abierto ampliamente, entonces se interviene para practicar la extracrión mannal de la placenta. Los peligros que en otros tiempos se anotaban a la rovisión uterina o a la extracción nanual de la placenta, han sido relegados wor la asepsia con que se procede y por la sistemática profilaxis del puerperio "ont antibióticos. Cuando existe la mewor duda de que hayan quedado restos l. Hentarios o cuando se aprecia la reteteion df membranas, se procede a la verisión uterina: esto da una seguridad 
mponderable porefue, repetmos, no ocurre la infecoión y sí a evitan las peligrosas hemorragias immediatas o tardías. Es más de temer la infección por retención de restos placentarios o de memhranas, que por la revisión uterina.

Como puede verse en los euadros, se hat praticado revisiones nterinas y se ha heroho extracoión artificial de la pla(*nta, sin romplicariones en el putrperio. La inciden.ia de las hemorragias en Jos $1 \cdot a<0 s$ que romentamos ha sido nula.

Duración del trabajo. Ya lo habiamos enunciado, la duración del trabajo ('n los partos espontáneos se ha bajado an forma impuesionante. Esto se dobe al sistema seguido: dilatación artificial [ie] anello para completarla en los rasos fádelles y a la ra-i sistemátiod prác. tica de la episiotomia en las primínara y a la episiotomia de neesidad en la multipards qu! anenazan deswarrare.

No nos detenemos en considerarionesibre las ventajas de la episiotomia sobre los desmarmos. ni a la ronvenien.ja de la episiotomía sistemation en las pri. miparas, por haber sido tratado esto tema en la tecis del doctor Oscar Mat. tinez Lemms. "El palde en las prims

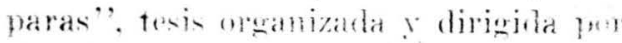

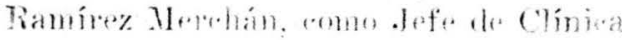
in tal éporat.

Puerperio. In indiee de ori d. infeceión puerperal sobre los 200 easos comentados. m 100\%, de éxito en la c.jratrizarion de las episiotomias y los descarros, así romo la normal evolución del puerperio en las intervenidas. constifuyen ma estadistica de lujo sobre la "ual mepere recalcarse con énfacice El fxito lo atribúmos semeillamente a los enidados seguirios en la Consulta Pro.
Jatal an alonde so proseroben los banos yaginales innecesarios, se tratan las cervicitis y los flujos en general y se advierten las normas rutinarias de higiene de la embarazada: a la extricta asepria de la atenoióm en las clímicas y muy particulanmente al empleo sistemático de antibirticos, en sn más alto porcen1aje la mutaliazina an en puerperio.

Tocia prierpera we trata romo si se injelara ma infecroún: se le administran, en promedio. 1.j gramos de sulfadiazina en forma continua, con una dosis inicial de 1.50 gramos $y$ con dosis parciales de 1 gramo cada 3 o 4 horas; th algumas oportmilades. elando ha babido extraceión manmal de la placen†A, dilatación artifivial del vello o desfarros irregulares. se rombinan las sulfax n la forma imlierala. ron ma o dos ampollas de penicilina an rehículo de aboreicin lenta a la dosis de 400.000 midades en ma sula inyecrión que se mpite 24 horas después.

Para asegurar ma lápida involución utarina, ma correcta eliminación loquial. $y$ para prevenir la posibilidad 4. hemiorragias tardías, a más de comremblar la integridad en la expulsión de 7a placenta y do lac membranas, se suministran diarianente 10 gotas de Me. thererin en dos dosi- diarias durante la formanemeia de la pareiente en la clími*a. Dosis mayores mo som aronsejables, no obstante que las indiradas por la casa productora son mayores, porque produ*n retraceión del enello nterino y retenrión de loquios.

la evallacion intestinal diaria se ar.erula por medio de lavados y laxan-

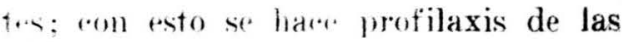
pielitis, no raras en a purperio, de parientes constipadas. 
El promedio de permanencia te las pacientes en la rlinica es de cinco días, cuando antes lo era de diez.

Anestexia. Dos han sido los sistemas de anestesia empleados: la general por inhalaciones intermitentes le reloprojano yoxigene, y la endovenoma general por pentothal ron inhalariones romeronitantes de oxigrno. Las inhalationes con diclo-oxígene son muy bien toleradas por las pacientes y no repereuten en forma apreciable sobre lid respiración fetal; los niños nacidos de madres anestesiadas por este sistema. respiran espontáneamente de manera immediata, siempre y ruando no se haya prolongado profundamente la anestesia.

En lo que se refiere al empleo del pentothal por via endovenosa, se exigen más requisitos y enidados que con el ciclo, para hablar en términos generales. las normas do sil empleo en partos nos han mierecido particular atención, ya que hemos sido de los más adictos a su empleo $y$, nuestras observaciones sobre el particular sirvieron de base para la tesis de grado del doctor Lueopoldo Rozo; en ella si expone la técnica. los resultados obtenidos y sus eontraindirarimes.

PORCENTAJES. ESPONTANFOS

$$
\text { Primigestantes: } 23=20 \% \text {. }
$$

Episiotomias: $\quad 9=39.13 \%$

Desgarmos: $\quad \because=13.04 \%$

Sin desgarro ni

$$
\text { episiotomia: } \quad 11=47.83 \%
$$

Multigestantes: $93=80 \%$

$\begin{array}{lrl}\text { Episiotomias: } & 1 & =2.16 \% \\ \text { Desgarros: } & 12 & =12.90 \%\end{array}$

Sin desgarro ni episiotomía: $\quad 79=84.94 \%$

Promedio de peso en partos espontábeos: 3.009 gramos.

Duración promedio de trabajo: 8 boras, i minutos.

\section{Medicación}

$\begin{array}{rlrl} & \text { Seidantes: } & 17 & =14.78 \% \\ \text { Ocitocicoss: } & 24 & =20.86 \% \\ \text { Inducidos: } & 6 & =5.29 \% \\ \text { Mixtos: } & 10 & =5.70 \%\end{array}$

Sin medicación: $58=50.44 \%$

\section{Presentaciones}

0. 1. A. : $90=78.26 \%$

O. D. P. : $20=17.40 \%$

O. D. A. : $3=2.60 \%$

1. I. P. : $2=1.74 \%$

Anestevia

(iillo:

$74=63.34 \%$

I'entothal:

$31=26.10 \%$

Pentothal-Ciclo: $\quad 4=3.47 \%$

Trileno:

$2=2.62 \%$

Sin anestesia :

$4=3.47 \%$ 


\section{(APTILA II}

FiRILPS

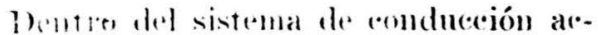
tiva det parto, para prevenir las compliearomes fetales. para prestar aten. rivin a radia pateiente tratando de sere.

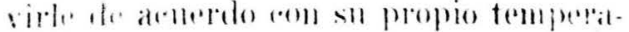

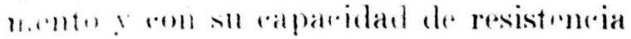
lisirat y de tolegameia del dolor y. ruande ex trabajo se prolonga por deficiencia es las fuerzas expulsivas. es compente. loy en día. terminar el parto (*on fórcops simpre y emando que estén presintes las rondiciones necesarias para su aplicación y que a conocimiento de la féconica. permita harerlo sin lesión fetal, ni mayor trammatismo para la madre. Como ya lo hemos enomentation no se espera la romplieación, sino que se evita. Esto se deduce de la eomsideración de la indi-ación: mient!as qua molas estadistivats hospitalarias. la más alta fre-nencia de aplicación corresponde al su. frimiento fetal, $11170 \%$, segín la tesis de grado del doctor Luis M. Medellín, en las estadísticas de este romentario (•asi no apareec la indicación por sufrimiento fetal. Que so hubiera presenta-

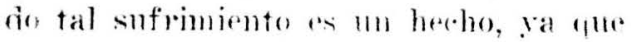
en 111 porrentaje anesiderable habia eir enlares del eordon y en otras, resistencia anomal del perine o insuficiencia do las romtraveiones para lograr la progresion normal del feto. De manera, pues. yule este procedimiento difiere de lo rláxieo en ese punto fundamental; 10 es el siffimiento fetal el que irmpone la intervención, simo mo homrado criferio de espereialista.

En enanto se refiere a las condiriomes neresarias para su aplicarión, que. dan enno norma ineludible las referentes a relaciones pelvifetales, al rue!lo

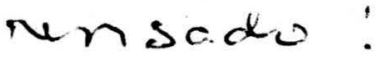

completanente dilatado o dilatable, al diagnóstico preciso de la variedad y de la posición de la presentación y a lo referente a la bolsa de las aguas; pero en lo que atañe a la altura de la presentarión. hablando entre especialistas y, coma requisito para ol técnico en esta rania de la medicina, no es indispensable el encajamiento de la presentación Y antes por el comtrario, la falta de enryjamiento pmede ser una de sus indiraciones y lo es de hecho como ha quedaclo probado en varias tesis de grado sobre el particular entre las que recordamos las de Cantera Durán, respaldada por la autoridad indiscutible del Profesor Victor Rodriguez Aponte y la (ii. Arenas Buenahora.

No es el adat de polemizar respecto a la posibilirad del fórceps por sobre el estrerho superior. pero como lo hemos visto aplicar. y lo aplicamos con éxito indurlable. poclemos decir que, asi como para quien no domina la especialidad ni la técnica equivaldría a "una embriotomía en feto vivo", para otros, es una sencilla y anodina intervención obste. trica.

Dos rasos sobre 4s estudiados aqui, fueron de aplicarión alta. La historia númiero 27. vaso número 2, es elocuente, no solamente $+n$ lo referente al éxito del fórceps alto en primípara, sino como respaldo de lo que hemos venido comentando :

Caso nimero 2. Historia número 27. Primigestante a término. Feto vivo. Inicia trabajo a las 0 horas de enero 7 de 1950. A las 2 horas 30 minutos, contratciones loves racla 5 minutos. Hace 20 horas que comenzó trabajo de parto $y$ las eontraceiones continúan cada $\mathbf{1 5}$ minutos. A las 16, de enero 7 d: 1950 , se completa la dilatacioin y se rompen 


\begin{tabular}{|c|c|c|c|c|c|c|c|c|}
\hline de orden & Historio & . PARIDAD & Present. & TRATAMIENTO PRE-PARTO & DURACION & $\begin{array}{c}\text { PETO } \\
\text { Sexo }\end{array}$ & ANESTESIA & OBSERVACIONES \\
\hline 3 & 28 & Secundiges & oinp & I amn Demerol & & & & \\
\hline
\end{tabular}

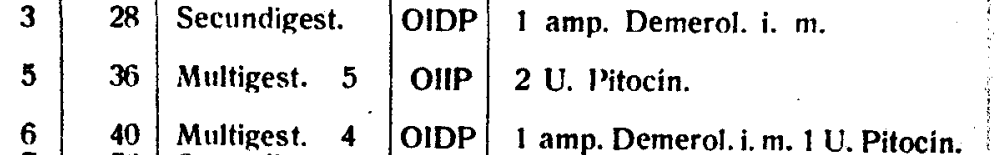

\begin{tabular}{ll|l|l|l}
6 & 40 & Multigest. 4 & OIDP & 1 amp. Demerol. i. m. 1 U. Pitocin. \\
7 & 50 & Secundigest. & OIA & 1 amp. Demerol. 1 cáps. Amytal.
\end{tabular}

67 Multigest. 3

Secundigest.

$18 \quad 114 \quad$ Primigest.

19118 Multigest. 3

OIDA 1 amp. Demerol. i. m.

OIA

OIDP $60 \mathrm{gr}$. aceite ricimio. Enema jabonoso. Dextrosa al $10 \%+3$

U. Pitocin gota gota.

23
28
28

\section{4}

$$
76
$$

103

$$
\begin{array}{r}
7 \\
8 \\
8 \\
8 \\
8 \\
9 \\
9 \\
9 \\
9 \\
9 \\
9 \\
10 \\
\\
103 \\
104 \\
100
\end{array}
$$

148 Multigest. 4

152. Secundigest.

187 Secundigest.

202 Primigest.

268 Multigest. 3

336 Multigest. 3

364 Multigest. 4

377 Multigest. 3

382 Secundigest.

457 Secundigest.

460 Multigest. 3

492 Secundigest.

512 Secundigest.

524 Multigest. 5

529 Secundigest.

OIA

2 U. Pitocin.

Demerol 1 amp. Nembutal 1 cap. Atrinal 1 supos.

OIIP Atrinal 1 amp. Trasent. 1 supos.

OIA

OIA

OIDP

2 U. Pitocin.

1 U. Pitocin.

2 suposit. Trasentina I amp.

OIA

Demerôt i. "m.

1 amp. Atrinal. 1 supositorio.

Trasentina. $1 \mathrm{U}$. Pitocin.

Media unidad de Pitocin.

I amp. Demerol. TU. Pitocín.

5 U. Pitocin.

597 Primigest.

6113 Secundigest.

620 Multigest. 4

Multigest.

626 Multigest.

658 Secundigest.

681 Secundigest.

683 Primigest.'

686 Secundigest.

\section{Multigest. 10}

757 Primiges:.

769 Primigest

$7 \times 1$ Secundigest.

822 Secundigest.

Q 30 Serundigest.

847 Multigest. 7 OIA

848 Nlultigest. 3

802 Primigest.

995 Primigest.

$\mathbf{9 0 5}$ llultigest. 3

101

o21 Nultigest. 3

OIDP

1030939 Secundigest.

104 1942 Secundigest.

106 949. Primigest

2 U. Pitocín.
2 U. Pitocín.

\begin{tabular}{|c|c|c|}
\hline .... & m 3000 & Ciclo $0=$ \\
\hline 6 horas & f 2800 & " \\
\hline $\begin{array}{c}11 \text { horas } \\
\ldots \ldots \\
10 \text { horas } \\
7 \text { horas } \\
\ldots . .\end{array}$ & $\begin{aligned} & \text { f } 3170 \\
& \text { m } 3500 \\
& \text { m } 3420 \\
& \text { m } 3120 \\
& \text { f } 2880\end{aligned}$ & $\begin{array}{l}\text { Pentothal } 0 \mathrm{~h} \\
\text { Ciclo } 0 \mathrm{z} \\
\text { Pantothal } \\
\text { Ciclo } 0: \\
\end{array}$ \\
\hline 12 horas & $\begin{array}{r}\text { f } \mathbf{3 4 n 0} \\
\text { m } 3000\end{array}$ & Fentothal \\
\hline $\begin{array}{c}10 \text { horas } \\
9 \text { horas } \\
\ldots . \\
\ldots .\end{array}$ & $\begin{array}{r}\text { f } 2700 \\
\text { m } 2800 \\
\text { f } 3560 \\
f \mathbf{3 0 0 0}\end{array}$ & $\begin{array}{c}\text { Ciclo } \\
\vdots \\
\text { Pentothal }\end{array}$ \\
\hline
\end{tabular}

branas.

2 circulares del cordón en el cuello.

Peq. desgarro perineal. Sutura.

Desgarro de primer grado. Desprendimiento en OS. 3 circulares en el cuello.

Extracción manual de la placenta. Desgarro pequeño. Ergotrate.

1 circular del cordón.

Ergotrate 1 amp.

Pasaron 300 c. c. de la Dextrosa. Hemorragia. 2 Ergotrates i. i. m. y otro endovenoso. Desgarro de primer grado.

\begin{tabular}{|c|c|c|c|}
\hline 5 horas & $m_{f} 2340$ & Ciclo & Daconara m \\
\hline
\end{tabular}

Desgarro mucoso.

30 horas m 3400

$\mathrm{m} 3000$

5 horas $m 3120$

… f 3450

… in 3000

Pentothal

Ciclo

17 horas f 2700 Pentothal

Ciclo.

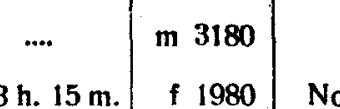

\begin{tabular}{c|c|l}
30 horas & in 3000 & Ciclo
\end{tabular}

$9 \mathrm{~h} .45 \mathrm{~m}$. f 2820 Pentothai

\section{.............}

Desgarro de primer grado.

Rupt. artificial de membranas.

Rupt. artificial de membranas.

Rupt. artificial de membranas.

Ergotrate 1 amp. Desgarro pequeño. Sutura.

Emb. de $7^{1 / 2}$ meses.

Se completa digitalmente la dilatación.

\begin{tabular}{c|c|l}
2 h. $30 \mathrm{~m}$. & f 3800 & Ciclo \\
f 3700 & Pentothal
\end{tabular}

Ergotrate. Se completa artifidalmente la dilatacion.

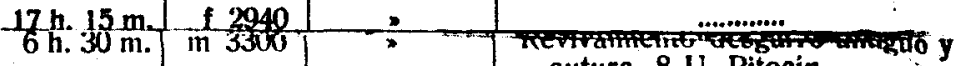
sutura. 8 U. Pitocín.

18 horas $m$ muerto Ciclo Episiotomia por cicatriz de des-

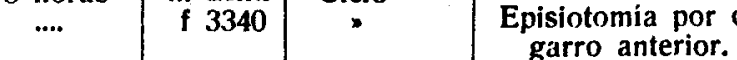

17 horas in 3300 Desgarro mucoso.

$6 \mathrm{~h} .30 \mathrm{~m}$. f 3000 Pentothal Se completa digitalmente dilatación. 8 U. Pitocín antes de alumbramiento.
1 amp. Mis. 2 U. Pitocín.

2 U. Pitocín.

1 amp. Demerol i. $m$.

1 amp. Demerol i. $m$.

2 U. Pitocín.

2 U. Pitocín.

OliA 2 U. Pitocin.

OIA

OIDA 1 amp. Morfina-atropina.

1 caips. Nembutal.

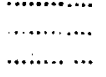

6 h. $45 \mathrm{~m}$. m 2860

2860

6 horas

4140

2 horas

f 3420

.....

m $3=00$

11 horas

f 3240

1 h. $30 \mathrm{~m}$. $\mathrm{m} 3700$

Ciclo y

Pentothal

Pentotha

Ciclo 02

$$
\text { ... }
$$

\section{...}

$$
\ldots
$$

3 horas f 3300 Pentothal
11 horas in 3400 Ciclo

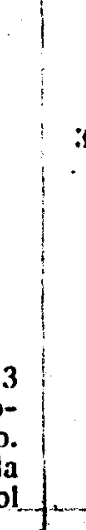
Atrinal.
3 horas $\#$ 3. 1 amp. Demerol

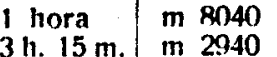

h. $30 \mathrm{~m}$. f 3160

3h. $30 \mathrm{~m}$. m 3040

h. $45 \mathrm{~m}$. f 3000

in 2940

h. $15 \mathrm{~m}$. m 3300

Pentothal Ciclo
No da tiempo para dar anestesia.

Se completa artificialmente la dilatación.

Episiotomia - bajo Pentothal.

Desgarro de primer grado. Shock. Circular apretada del cuello. Hemorragia por inercia uterina. 2 Ergotrate endovenoso.

Rupt. artificial de las membranas.

Episiotomia. 2 circulares en el cuello.

Ruptura artificial de membranas. Desgarro superficial.

Se completa artificialmente la dilatación.

Episiotomia. Ruptura artificial de membranas.$$
\text { ................ }
$$

Se completa artificialmente dilatación. Ruptura artificial de membranas. Circular del cordón en el cuello.

Ruptura artificial membranas. 


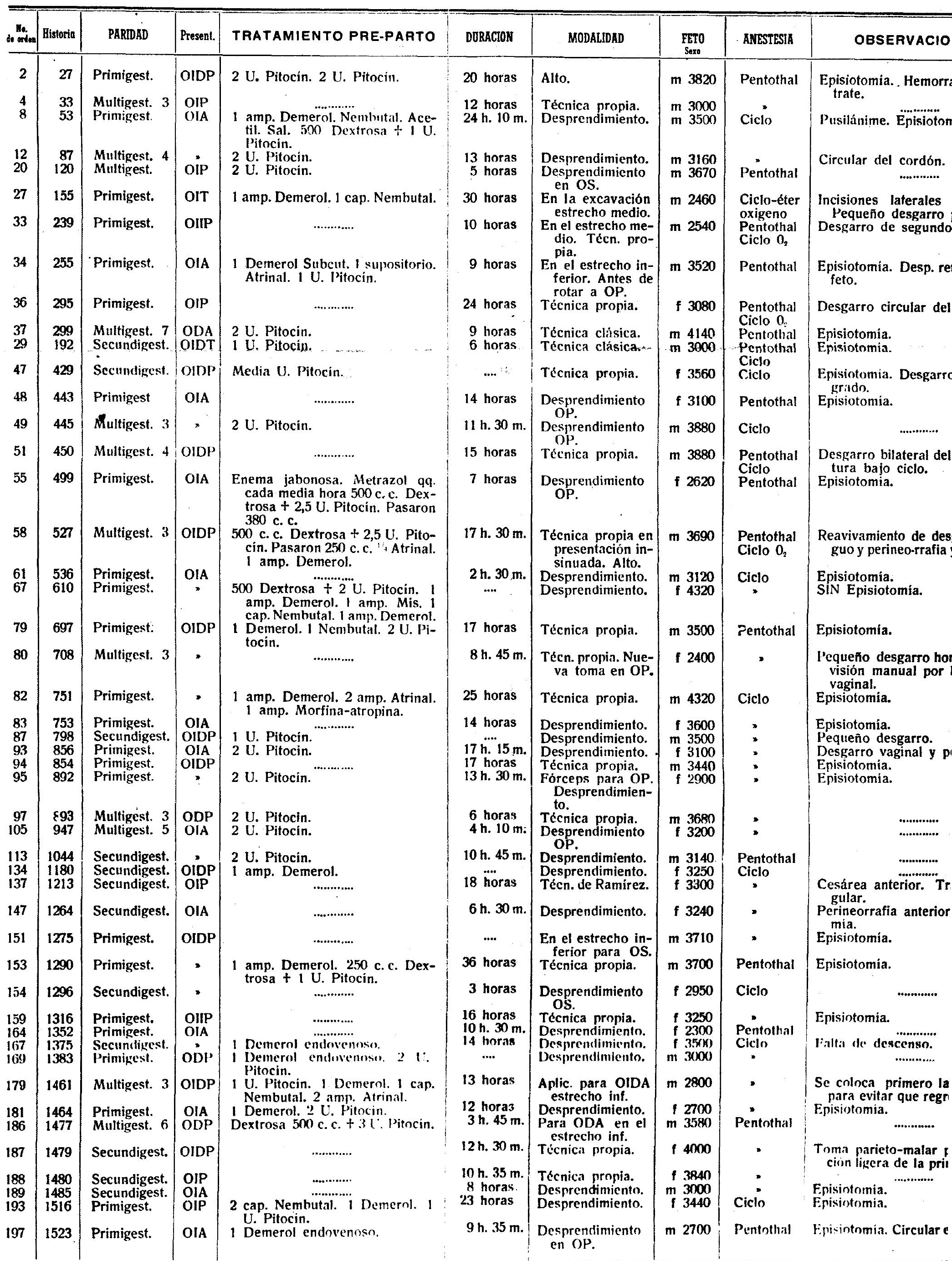


13. Pitocin.

2 U. Pitocin.

\section{U. Pitocin}

Induccion: Lallado evacuador. 30 gr. aceite ricino. Foliculina de

1 U. Pitocin. 2 U. Pitocín.

1367 Multigest. 3

68

1377

Primigest.

1400 Primigest

1404 Primigest.

1412 1414

Multigest. 3 Multigest. 3

1419 Secundigest. 1420 Multigest. 4

1434

Multigest. 3

Multigest. j

Secundigest.

Multigest. 3

Secundigest.

Secundigest. Multigest. 3

\section{Secundigest.} Secundigest.

Multigest. 3 Secundigest.

Secundigest.

Multigest. 3 Secundigest.
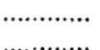

1 cap. Nembutal. 1 amp. Demerol endovenoso. 2 U. Pitocin.

\section{OIDP \\ 2 U. Pitocín.}

1 U. Pitocín. 1 U. Pitocín. 1 cap. Nembutal.

OIDP OIA

1 cap. Nembutal. 1 Demerol i.m.

1 amp. Atrinal. 1 Demerol endovenoso.

1 caps. Nembutal.

2 U. Pitocín.

1 Demerol endovenoso.

2 U. Pitocin.

1 Demerol endovenoso.

2 U. Pitocin.

3 U. Pitocín.

Inducción: Aceite de ricino 60 . $10 \mathrm{mg} .1$ amp. Dextrosa 10\% +3 U. Pitocin. Metrazol qq. cada '/2 hor.t hasta 3 tabl. Pitocin $2 \mathrm{U}$, hasta $6 \mathrm{U}$. cada hora.

Ciclo m 3900 Trilene

im 3500

Ciclo

m 2190

\begin{tabular}{l|r}
6 h. $30 \mathrm{~m}$. & m 3000 \\
f 3500
\end{tabular}

Trilene

Pentothal

Pentothal y

Ciclo

10 horas

f 3360

Rempira stificial de membranas.

Episotomia. Sutura en 2 planu. , ..............

puisivo.

Ruptura prematura de memlra nas. Episotomía.

Ruptura artificial de membranas.

Ciclo y

Pentothal

10 horas

f 2600 Ciclo

12 horas

m 2900

$9 \mathrm{~h} .30 \mathrm{~m} . \quad \mathrm{m} 3700$

14 horas f 3100

12 horas

....

f 2880

m 3000

Pentothal

Ciclo

23 horas

m 3160

m 3500

"

Pentothal

m 3260

Ciclo

5 horas Mueito y

macerado

m 3000

m 2840

....

f 3100

Pentothal

4 horas

m 3040

f 3440

Ciclo

Pentothal

2 horas

m 2700

2 h. $30 \mathrm{~m}$.

m 31$) 60$

Fentothal

2 horas

m 3360

m 3640

$\cdots$

f 3360

7 h. $15 \mathrm{~m}$.

m 3900

Pentothal
Desgarro mucosa vaginal -para las suturas-. Hemorragia.

Episotomia. Doble circular del cordón. Alumbramiento artificial (manual).

Desgarro de segundo grado.

Ruptura artificial de membranas. Desgarro de mucosa. 1 circular del cordón.

Ruptura artificial de membranas.

Ergotrate 1 amp.

Espasmo de la laringe que cede con 1 amp. de morfina-atropina.

F. M. por nudo verdadero del cordón.

Ruptura artificial de membranas.

Se completa digitalmente dilatación. Ruptura artificial de membranas.

8 U. Pitocín después alumbramiento.

5 U. Pitocin antes de alumbramien-

Ruptura artificial de membranas.

7 U. Pitocin post-alumbramiento. 1 amp. Ergotrate i. m.

Ruptura artificial de membranas. Desgarro.

Ruptura artificial de membranas. to. Desgarro de la horquilla. 
la presentación móvil. Se pasa a la sala a las 19 horas y bajo anestesia se aplica fórceps por encima del estrecho superior, previa aplicación de 2 unidades de Pitocín. Se hace nueva toma ell el estrecho inferior para desprendimiento y se obtiene niño que respira espontáneamente, de 3.020 gramos. AlumbraJniento espontáneo. Hay ligera hemorragia y se aplica una ampolla de ergotrato endovenoso.

Anestesia: Pentothal 20 centigramos. I)rración : 15 minutos.

Antes del desprendimiento se hizo episiotomía que se sutura con catgut.

la sencillez y facilidad de la intervención se deducen muy claramente de Ja observación de la anestesia empleada, d. su cantidad $y$ de su duración. Pentuthal 20 centigramos, es una dosis mínima y una duración de 15 minutos, indica que la intervención no turo complicación. Los resultados fetales no hay para qué comentarlos si tenemos co cuenta que en conjunto de los fórceps, no hubo ni un solo caso de mortalidad fetal ni de morbosidad de importancia; Jos niños nacieron vivos y salieron en perfectas eondiciomes de la clínica.

El "aso número 58 es, por decirlo así, un resumen de la manera de obrar por lc vial la transeribimos en lo pertinente, llamando la atención sobre la combinarión de indicaciones y sobre al conjunte de la observación, en donde se ve muy aparentemente cómo una inercia primitiva en una multípara, logra corecricke hasta dar ma areptable dilatación cervical, pero no el encajamiento.

(ase número 58. Historia número 527. Multigestante a término. Feto vivo. Parai III.
Marzo 2 de 1950. Hora: 17 y 15. Duración: 17 horas y 30 minutos.

El 19 de marzo comenzó a sentir dolores a las 23, pero tenues y espariados; a las 5 a. m., del 2, llega a la cliniwa y (*omo no se observa trabajo frances se ordend indur.ion : Pitorín $21 / 2$ midades en 500 (r. e. de dextrosid al 10\% re de los (ine alranzaron a inyectarse 250 r. c., Cescle las 11 hasta las 14; 001 esto se deseneadena el trabajo. Se le hace tacto vaginal a las 12 y 30 que revela: cuello borrado, duro, dilatación 4 centímetros, bolsa íntegra, presentación por encima del estrecho superior.

Se apliea nund ampolla de atrinal, a las 13 horas. Siguió con buen trabajo, contracoiones cada 4 minutos y 30 segrundos de duración. A las 14 y 30, nuevo tacto vaginal: cuello borrado, duro, resistente, dilatación de 5 a 6 centímetros, presentación en OIDP, por encima diel estrecho superior. Se ordena una ampolla de Demerol intramuscular. $\Lambda$ las 16 y 30 , se le practica nnevo tacto vaginal yne revela: cuello borrado, blando. aasi completamente dilatado, presentación OIDP, por eucima del estrecho superior. Bolsa rota. Se pasa a la sala y bajo anestesia se completa digitalmente la dilatación rechazando el horde anterior del euello y se aplica fóreps con presentación insinuada en

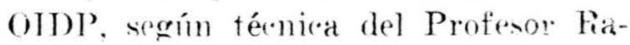
mírez Merehán, asimilándolo a ODA; encajamiento. descenso y rotación fáciJes; toma ideal. A las 17 y 15 se extrae un niño de 3.690 gramos que respira espontáneamente.

Alumbramiento artificial a los 20 minutos (manual). 
anenesus: rentothal (1.s) rentiglamos. 0.2. para la aplicación del fóreeps Y r.joppropano-0.2, para la perineorrafia ron miorrafia de los elevalores, despmés de reavivar clexgarro antiguo.

El procedimiento seguido ya ha side objerta de comentario. la terminarion

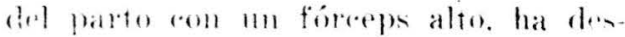

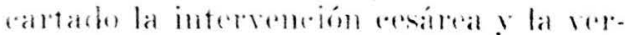
som bor maniobmas intermas. de la ellal somos ada día menos adietos. La andotesia eon pentothal indies. entre otras ersals. que ol partero previó la farilidad y rapidez de la intervención, ya que dicha anestesia no es acomsejable en forma prolongada ni amu euando el desprendimiento se espera para 1 lu lapo sio menor de 15 a 20 minutos. Despué fe teminado a parto se practico pe. rineorrafia rom miorrafia de los eleradores para desgarro antiguo, lo yure demuestra las buenas condiciomes de $l_{a}$ paciente que tolera tal intrrvención sobreagregada al parto; entonces se com. jeté la anestesia con eiclopropano-(1); la anestesia anterior con pentothal. ha rervido de inducción a esta última.

Xo son más que dos observaciones. pero notese gue vienen sobre un total de tr fórceps, lo que da un indice de $4.16 r^{\circ}$ en las intervenciones de un año. Y romo no es mid novedad tal intervencióm robran mérito domostrativo los casos en enestión.

Ta primiparialad o la multiparidad so son en manera algma despreriables pard el efereto de luma ronducta; muy jer el eontrario han de tenerse siempre en enenta. Mayores posibilidades d. erar poporeionan la primipara que la multipal'a asi romo mavores facilidades y menos complicariones son de esperar en esta hiltima. Pero ese es tan sólo un faxtor al ellal hay que darle su justo ralor: sin arenturarst en las multíparas a intervenciones inconsultas $y$ sin rehuir la terminación por las vías naturales en la primipara, por al mero hecho dis serlo.

En las aplicaromen de este comentat. rio, hay 1018 so, de primigestantes y m sor, de multigestantes; si se tiene (i) (ellental que en las primiparas se sigre asi de lutina la aplieación profi. lictiva de desprendimiento como se hace también la episiotomia. se deduce que (a) las multiparas hav relativamente 1:ayor número de aplicarión del fórceps por otras eausas ya sed por inercia 0 jor aeortar, sin detrimento alguno, la dillarion del trabajo.

Aplienciones de desprendimiento. Casi sistematicamente se sigue en las primiparas la conducta de desprendimiente con fórceps y episiotomía. La mayoría do las veces el fórceps se ha aplicado en toma directa en el estrecho inferior, prer.isamente para apresurar el desprendimiento, con la paciente bajo anestesia. Se evitan en tal forma los esfuerzos del desprendimiento que a más de ser pemosisimos para la parturienta rolocan al partero en la inerte actitud de ponerse por delante para aconsejar a su pacientr que "puje"... Cuando la prosentación abomba en el periné. se retiran las rucharas del fóreps y s termina la expulsión eon episiotomía. En lo que a ésta se refiere, siempre se ha practirado una episiotomía mediana ligeramente desviada havia un lado a partir de la horquilla; "s preeisamente una rpisiotomía que imita la vía seguida por los deserarros pero ron la imponderable ventaja de que previamente se controla su extensión. su recrularidad y que no es una herida trammática sino una heri- 
(a qullirglina la glir mego se va a reponer.

No solamente por el estudio de las historias clinicas, sino por la diaria observarión en la Clínica de La Magdalena - purele afirmal que es man ppisotomía di. grameles rentajas por ellanto nuncad se ha prolongado, al despremelerse el feto, hasta interexal el esfinter y ha prendido en el reiento por ciento de los asos. Sil reposición se have en tres pla Jos: mono nucoso, ofro de fascia y músculo y un tereer plano de piel; se usa para todas la misma sutura : catgut simple numero 1 o 2 . Cuando ha sido muy regular la herida y fácilmente afrontable, so ha practicado una sutura continua. monsejada por De Lee que se inicia en la murosa raginal en donde se aeja reparado el abo corto del primer punte: matez rerrada la mueosa, se continua con el plano múseulo-aponeuróticos. - implere en forma continua; una vez llegado al final de la herida se devuelve 'n busc.d del cabo reparado, afrontando la piel con sutura intradérmica y :reminando donde se había empezalo.

Puede verse on el cuadro correspom-

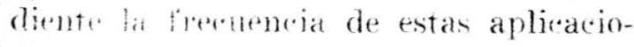
nes, a a como las variedades de despresudimiente el porentaje de las apisiotorufias. Lama la ateneión el porentaje de despartes produridos en apuellas pafienter an las ylle un periné elástico haeia pensar en lo inofirioso de la episiotomia.

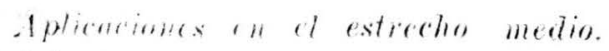
Originalumente la mayor incidenuia de apliedrones del foreeps rexaía sobre

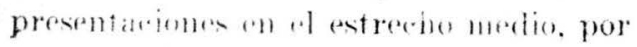
que rumplido of requisito del encajamiento y hahiendo dado larga a la evolu-

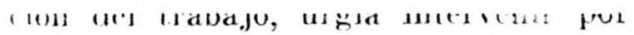
sufrimiento fetal o por agotamisuto de la parturienta o por ambas cosa cuando no por óbito fetal. Tales aplicariones d. angustia más que de urgencia eran las únicas permitidas por la ortodoxia wistétricas.

Desde hergo, el porcentaje de murtalilad fetal tenía que ser alto y. como am hay quienes tienen ese criterio sobre ol

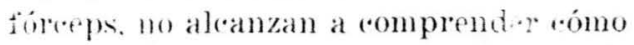
puede presentarse una estadísti at más (1) menos (opiosa en aplicaciones sin que aparezea $m$ alto porcentaje de desastres fetales. Pero la razón es muy clara: En primer lugar se aplica el fórcep segin criterio informado $y$ honesto d.el especialista sin que se vea la urgen.ia do hacerlo; en la mayoría se trata a apli-aviones profilácticas; $y$ en el stomndo lugar la vigilaneia enidadosa y la eomflanza en la intervención llevan a harerla oportunamente y a sabiendas de lo cue se va a practicar y de lo qur va a ocurrir, dentro de la limitación natural de los conocimientos hmmanos y in tal forma, los resultados son necesariamente buenos.

Las aplicaromes en el estrecho medis (n) los rasos que romentamos tatn (o)sido $107.50 \%$ del total de la- aplicarones, dejande el 58.34\% parat las del estrecho inferior y $4.16 \%$ para las rel estreeho superior.

las variedades sobre las cuales se han aplicado, aparecen en los cuadrie adimotos; llevan la primacía las apliearejones sobm el diámetro oblicuo izunier(?), como era de esperarse.

Técuicas. Se ha seguido la térnica Hasiea en las tomas directas y re las tomas obliellas de varielad anteriul. poryue en las transversas y en las oblievas

\section{$138 \quad$ Obstetricia y Ginecologí}


de variedad posterior, se ha seguido la técnica original del Profesor agregado Ramírez Merchán, que sirvió de tema de tesis al doctor Isaías Arenas Buenahora r que mereció el discernimiento de mérito por el Jurado de Jueces de Tesis nn consideración a la utilidad de tal pro(...himianto.

El tireeps empleado ha side siempi. e. Simpon corto.

\section{FORCEPS}

\section{Altura}

En el estrecho superior: $2=4.16 \%$ En el estrecho medio: $18=37.50 \%$ En el estrecho inferior: $28=58.34 \%$

Primigestantes: $24=50 \%$

Multigestantes: $24=50 \%$

Desprendimiento en:

o. S. $3=1.07 \%$

O. P. $25=98.93 \%$

\section{Anestesia}

$\begin{array}{llrl}\text { Pentothal : } & 17 & =35.41 \% \\ \text { Pentothal } & & & \\ & \text { Ciclo: } & 5 & =10.41 \% \\ \text { Ciclo-02: } & 25 & =52.09 \% \\ \text { Cielo-éter-02: } & 1 & =2.09 \%\end{array}$

Varicdades:

En O. I. A. : $18=37.50 \%$

En O. D. P. : $20=41.66 \%$

En O. I. P. : $7=14.58 \%$

En O. I. T. : $2=4.18 \%$

En O. D. A. : $1=2.08 \%$

Técnicas:

Directas rlásicas: $28=58.34 \%$.

Oblicuas: 20.

Clásians : $5=10.41 \%$.

Técnìn Ramírez: $15=31.25 \%$.
Promedio de peno en foreeps: 3.256 gramos.

\section{Mederacion}

\begin{tabular}{|c|c|}
\hline Induridos: & $\bar{i}=10.41 \%$ \\
\hline Sirdantes: & $\bar{s}=10.41 \%$ \\
\hline Oeitórieos: & $13=27.08 \%$ \\
\hline Nixton: & $=12.52$ \\
\hline Sin merlicarion & $19=39.55$ \\
\hline
\end{tabular}

$$
\text { Evixiotumin: : } 21=4: 75 \% \text {. }
$$

En primigestantes: $17=00.95 \%$

En multigestantes: $\quad t=20.05 \%$

Desgarros: 7 .

En primigestantes: $4=57.14 \%$

En multigestantes: $3=42.86 \%$

Sim desgarro ni episiotomía: 20.

En primigestanter: $: 3=15 \%$

En multigestantes: $17=85 \%$

\section{CAPITLLO III}

CESAREAS

El índice de incidencias de la operación cesárea, ligeramente superior a un $4 \%$. es un índice alto que se expliea por las razomes que impusieron la interven. ción, menos que por ma relación de estadistira. En efecto, no hay entre nosotros ma tendencia intervencionista por la vía alta ni mucho menos. El hábil manejo del fórceps, su indicación en algunos casos en que parece desplazar a la cesárea y, la prudencia en el uso de ocitócicos, son factores gue contribuyen a quitarle preponderancia it la casárea. P'ero euando surge su indicación como insustituíble, se practica con gran confianza porque tiene mínimos peligros y porque el porvenir obstétrico de la par- 
tivienta no queda comprometido, desde gue se siga la térniea de la resínea segsisentaria.

Ina residea segmentaria no impone la terminación ulterior de otros embarazos por cesárea, sino en el caso en que fo repitiera la indicación de la priniera 6ersistieran sus motivos, como an las estrerbeces pélviods o en las deiomata ciones de la matriz o en la perturbacion sistemática de la dinámica uterina. Purque ruande una resárea ha siclo indicacia por und causa que no ha de repetirse, no hay por qué temer del parto por las vias naturales, debidamente conducid, y vigilado.

Para respaldo de la afirmación inicial de que el indice alto se explica menos por una relación de estadística yue por coinciblemeia de indicaciones inespuivables, merere atención el hecho de cule no primó la incidencia de primiparas sobre multiparas; en efecto sobre ocho interven.iones cuatro recayeron sobse primípara y cuatro sobre multíparas, pero no distribuídas caprichosamente: una de las viatro primíparas, la de la historia nimero 1.2.21, “aso número 1:3!), fue intervenida (ne embarazo de 8 meses por ruptur: prematma de la bolsa de las agnas thipertomia uterina, en ronsideración a yor esta padeiente, ostentoramente hipertiroideand, llevaba 15 anos de esterilidad o de infereundirad y no cra permitido esprar el aleatorjo desenlace de un parto espontáneo en una paciente que inicia trabajo de parto eon una rupitura prematura de la bolsa. con hipertonia uterina y sin contraceiones, a más de you su trastorno endocrino anforizaba a pensale en una posible irrefrularialad del trabajo. Quién se atrevería a negale razón a la intervención en este "aso? Y sin embalgo, no ellatra runtro de Jas indiraciomes rutinarias de lis libros.

()tro caso, el número 176, histuria nunero 1.4:30 de primíparas, rorresponde a nna señora de 34 dños en quilin, a pesar de eontraceiones definidas y rom sufiejente prueba no hubo eferto sobre e] raello nterino. Se iniriaron has ent-

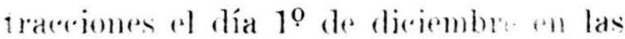
loorate de la mañana; Moró todo e. dia ? la noche "n trabajo irregrular: elia : lubo trabajo definiclo eon bnenas ermtrareiones pero el enello permanecio borrarlo y entreabierto; la presentación insimuada en O.IA. No había devpoporción. Se trataba, pues. de ma perturbarión en la dinámjea uterina. At nua disistolia sin sinergia funcional. pur lo domáx nada rara en las primiparas vie jas. El peso del niños que tind de 3800

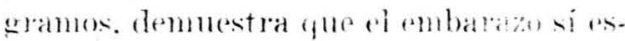
taba a término y de consignirnte. no rabe dudar respereto a la real y idtinida prepturbación dináming y no a nua sim. ple iniciación preeo\% de contracejomes literinds remediable con sedantes y aspera.

La tercera de las primíparas, ran múmero 152. historia múmero 1.2-6;. (.)rresponde también a mona inereia primitiva, a nma oligosistolia primitiva smergia fomejomal. J)iec trextmaluente la observacion:

Inicio trabajo a las 8 horas ate 2.2 do ortubre. en forma irregular y epaciada; en la norhe durmió bajo lat areión le nembutal 0,20 cotrmgs. ; el 228 -igne el tlabajo irregular; en la nochu der.mene. El 24 en la mañana se rempie?en altifjeialmente las membramas y al tacto vaginal se eneuentra: rnello borrato, elltreabiorto y presentarión en la rxeatración. Se lo apliea una ampolleta de a alElmpmina $y$ se le administran :) rom- 
primidos de metrazol-quinind aumentan las contracciones en intensidad $y$ frecuencia; en la noche el trabajo se hace irregular. El 25 de octubre a las 9 el tacto reveló las mismas condiviones d. Jos dias anteriores por lo cual se inicrvino con cesárea segmentaria transpevitomeal por inereid primitiva. El niño fesal 3.900 grames. indiee ererto de perforta madmere Tyes dias de espera y

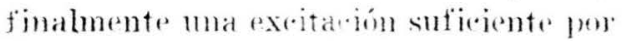
ruptura de la bolsa y"administración de derivados de la quinima, sin respuestat oficiente, justifican al máximo la conducta seguida.

El lapso de espera tan largo se explica por dos razones: la primera, porque no había ni agotamiento de la pariente ni sufrimiento fetal, y la segunda, porque nunca se decide precipitadamente una cesárea si no existen las condiciones e indicariones de urgencia que así lo impongan. Esto para demostrar que no hay una tendencia intervencionista en tal sentido.

Finalmente la cuarta de las intervenciones, comentadas no en orden de su ciecución sino más bien en orden de intorés, es el caso número 107, historia número 960 que se praıticó también por ma disistolia sin sinereia funcional. 1)"zante dos dias estluw an trabajo irreqular y en observacion; el feto fue do puso inferior al del término, no obstante estarlo según la fecha de las últimas reglas que daba la pariente; pudo tratarse de una iniciación prematura del trabajo, pero en todo vaso hubo trabajo

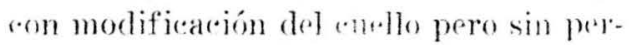
mitir el parto por las vias naturales.

Resumiendo las indiraciomes ate lats "natro interveneionse primíparas vemos cómo las vuatro fueron por causa de la dinámica uterina perturbada. Si bin es cierto guc hay factores predisponcutes que no pueden despreciarse, como la infecundidal de 15 años eir una y la edad en la otra, la determinante fundamental fue la oligosistolia o la disistolia que no permitiendo la dilataciom del evello, hacian imposible el parto por las vias maturales. Pretender instifiear ofras eonductas sería neein nor Io rual eortanos el comentario. Val. j chservar que no se practicó ningma de estas intervenciones por causa de estrechez o de desproporeión cefalopélvi(a) podría argüirse, de acuerdo con ideas clásicas, que puesto que fue factible la cesárea las presentaciones no se habian encajado y, romo es regla que "n las primíparas la presentación se enraja en la última quincena del embarazo, en éstas debía haber desproporeión. la respuesta la da la experiencia: no or rierto el encajamiento de la riltima quincena en las primíparas; el denominado encajaniento sin previo trabajo, no lo es de verdad; y no impide la resárea el herho de que se haya producido.

Pasamblo a comentar las cuatro cesáreas revaidas en multiparas, min de tereera, en pariente estrecha con bnena fresentaleión y una de segunda también

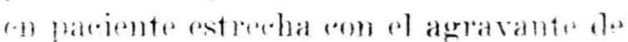
fuesentarion de hombre y eon al ante-

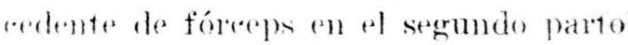
veil foto muerto. Otra de las cuateo. fes mat multigestante 111 eon anteredentes cbstétricos normales pero en la que se impuse la interveneión por la dohle indieareión de plarenta previa y presentarion de hombro. Ex sabide que an el - -tade actual do la obstetriejat, para pecolver bos casos de plarenta prexia. prima la interveneion wesírea aun en los cases de foto no viable desile que la lare vorragia sea ropiosa, o sin serlo. des- 
iie que se trate de placenta central. Finalmente hay un caso en que la interrención fue impuesta por las circunstan-ias especiales a que llegó la paciente, después de haber sido estimulada con w.itóli.os en la forma expuesta anteriornent. y en la que queda la sospecha de latber mocedido un poco a la ligera en ei live de los ocitócicos por cuanto se trataba de una presentación de pelvis $y$ habia el antecedente de una presenta. ción de hombro. El resumen del caso pernite formarse un juicio al respecto por lo cual se transeribe.

Cåo número 32. Historia número 335. Multigestante a término. Feto vivo Para III.

Se le hace tacto vaginal a las 19 horas del 21 de febrero de 1950: cuello sin borrar, dehiscente; bolsa íntegra. Presentarión de pelvis completa. Contraceiones irregulares.

Febrero 22 de 1950: a las 7 horas se induce el parto con 3 unidades de pitocín an 500 e. (e. de dextrosa al $10 \%$ gota a gota endovenosa. $A$ los 10 minutos se aprecian contracciones tenues que son irrogulares, termina el suero y ce. san también las contrariones. A las 15 horas aplican $500 \cdots$ a de dextrosa, al $10 \%$ : 5 unidales de pitorín ; reaparecen lits contraceiones un poco más fuertes. $A$ las 19 horas durante una contracoion se rompe la bolsa; sale abunclante lípuido amniótico normal; hasta alpli s: le han inyectado $400 \mathrm{ce}$. desturo: de suspende éste y se have tacto vaginal: "hello sin borrar. dehiscente, sílo deju parar un dedo, bolsa rota. Presentalion de peivis completa. Al supri- nir el suero desaparecen las contrasciones y se ordend un nembutal-acetilsal. Durante la noche hubo pocas contrac(riolies.

$A$ las! 930 horas del 23, tacto vaginal: procidencia deel cordón. Presenta. win de hombro " hipertonía uterina.

\section{iNTHW Y}

Febrero 2:3 de 19.0. Hora: 11 a. m. Duración: 1 hora.

Cesárea segmentaria transperitoneal areiforme por presentación de hombro, con procidencia del cordón e hipertonía uterina. Bajo anestesia general eon ciclopropano-02 y previa induceión de ésta con pentothal ( 0.10 gramos) laparatomía media infraumbilical. Histerotomía por disociación arciforme del segmento inferior. Se presentaba el hombro derecho, extracción mediante versión. Se inyecta pitocin en el miometrio. Alumbramiento normal.

Reposición del útero en tres planos. En el ángulo izquierdo de la berida antes de cerrar peritóneo, se deja una mecha-tapón de oxicel. Laparotomía cerrada en tres planos. Piel con ayraffes. La niña respiró espontáneamente y pesó 2.700 gramos.

N. B. La presentación originaluente de pelvis se modifié por el efecto del pitocín de la indución que desencadenó contracciones indoloras e inetectivas sobre el cuello, las que produjernu ruptura prematura de la bolsa y mís tarde, procidencia del cordón umbili al

Anestesias: Pentothal-02 Ciclopropano-02.

Tal es el resumen de las intervencioues cesáreas en lo que concierme a la indirarión, a las razones que la impu- 


\section{$C E S A R E A S$}

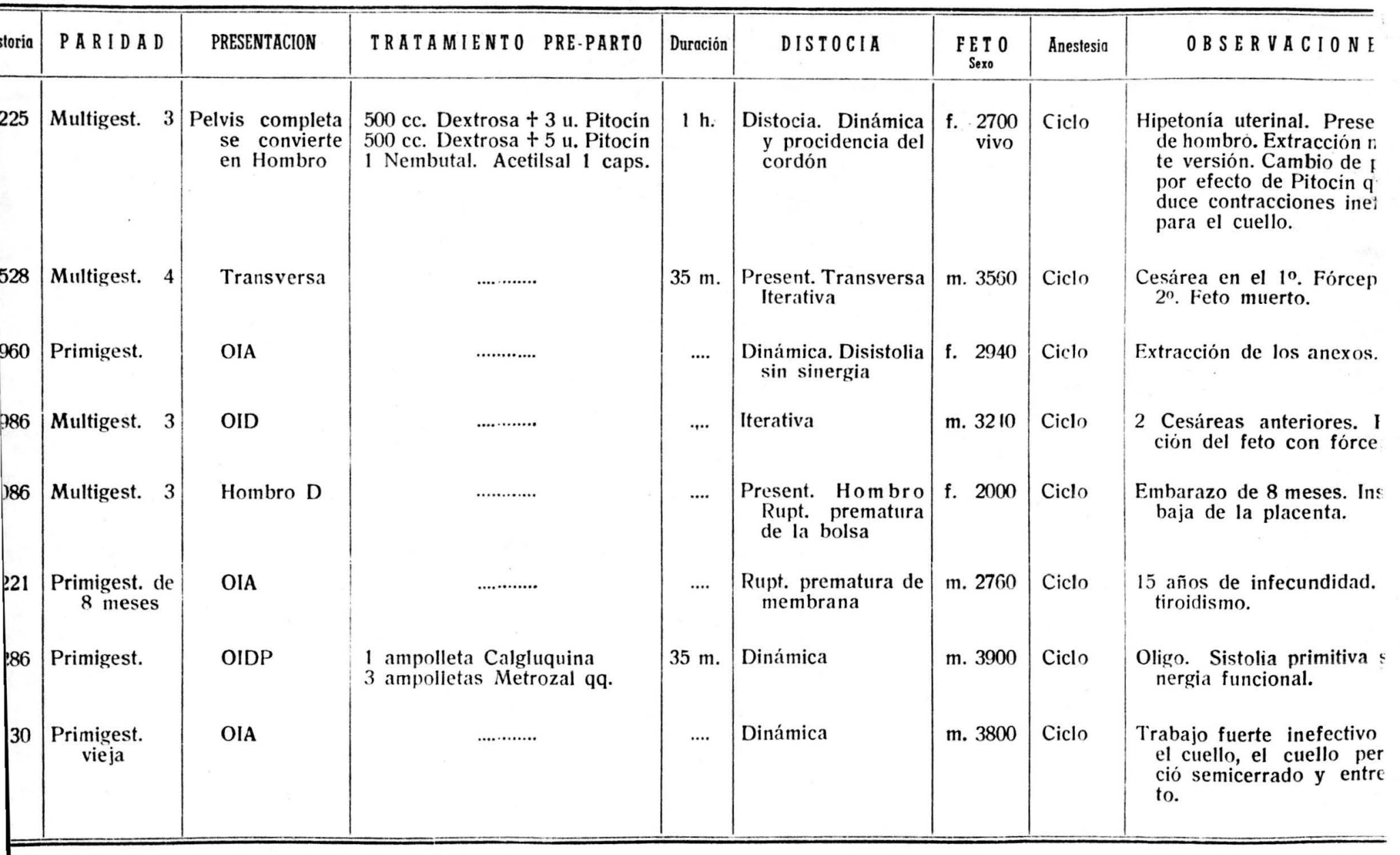


sobre il total de casos obstétrien que fanms estudiando.

El proultado final ha sido de éxito (in el 100): de los rases porque no ha habj(n) ni morbosidad ni mortalidad materna "fetal y porgue la reposición de las parrientes se ha hereho en lat lapso medio d. 10 días.

la tereniea seguida ha sidu sicinpre la de la resárea segmentaria transperitoneal ron disociación arciforme del segmento Y la anestesia empleada, la general con ejclopropano-oxígeno. Tordas han sido cesáreas más o menos puras y se ha her-ho profilaxis de la infeceion en la forma mencionada antes.

Como se anotó al principio, el índice de incidencias es alto, si de manera abstracta se lo compara con otros; en efeeto, entre nosotros, según la magnifica tesis del doctor Gonzalo Echeverry, el inclion de incidencias en la clientela hospitalaria es del 1.99\%, índice lograrlo sobre un total de 1.997 partos. Pero en estadisticas modernas hay índices inferiores y los hay también que supiran los ya mencionados. Para respaldar to dicho, transeribimos lo siguiente, tomando de im documentado artículo de Rohert Kitzner, publicado en el número correspondiente a enero del presente año. A American Journal of Gynecolog!l and Obstetrics: "Hennes: noted that in his elinie the incidence private rases was 5.82 per cent against 1.75 per "ent for service eases. Mc Cormick Found the ratio to be 8.80 per cent private and 2.80 per cent service. Is there justification for this marked diffuren.e. : Do social position and income l,racket influence the decision of the obstetrician? Is a greated premium plaand on fetal life in private patients? matermal soft parts traumatized and prolonged labor condoned so that interns and residents may, by trial and error. observe and rommit obstetrical mistakes as part of a recognized tea(hing program?

The answers to these questions may be obtained only by a study of a large series of cases plotting fetal mortality and fetal salvage rate against cesarean section and operative delivery. D'Esopo has racently presented excellent stadistical material in this regard".

TABLE I CESAREAN SECTION RATES \%o

New Have Hospital . . . . . . . . . $\quad 5.9$

$\begin{array}{lllllll}\text { Margaret Hague . . . . . . . . . } & 2.6\end{array}$

Chicago Lying-in . . . . . . . . . . 2.5

New York Hospital .. . . . . . . . 2.1

Boston City Hospital . . . . . . . . 4.2

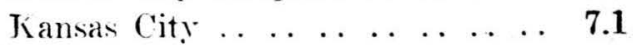

Johns Hopkins Hospital . . . . 5.5

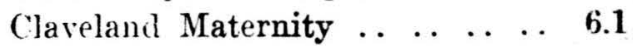

Philadelphia Lying-in . . . . . . . 5.8

$\begin{array}{llllllll}\text { Sloane Hospital } & \ldots & \ldots & \ldots & \ldots & \ldots & \ldots & 5.8\end{array}$

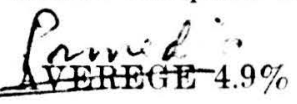

Cincinnati-Bethesda. $1940-1949 \quad \ldots \quad 1.3$

Cincinnati Christ. 1940-1949 . . . 1.6

Cincimnati-Good Samaritan. 1940$\begin{array}{lllllllll}1949 & \ldots & \ldots & \ldots & \ldots & \ldots & \ldots & \ldots & \ldots\end{array}$

Cincinnati Jewish. 1940-1949 _. . . 2.6 I.

AYRAGE $1.8 \%$

Cincinnati General. 1940-1949 .. 1.0

Tal parece que es de universal aceptación la injustificable conducta de proceder de una manera en clientela particular, y de otra en clientela hos- 
inalaria, cuando no debiera hacesse usf.ren.jación alguna, si se está obrando homrada y científicamente. Como tenemos la eonvicción de que en la clientela yarticnlar razones irrefutables imponen Jas actuaciones, es preciso procurar que la rlientela hospitalaria se beneficie de ellas en igual forma.

\section{CAPITULO IV}

\section{ABORTOS}

De 22 historias clínicas referentes a abortos, hubo 7 en los cuales se estableció tratamiento médico de rutina, (c)n resultado solamente en 3, que figuran como simple amenaza de aborto; en las 4 restantes, fue inefectivo.

Descle que no se pueda precisar la causa productora del aborto, resulta poco menos que imposible obrar en for. ma segura. El tratamiento de rutina de las amenazas de aborto, se reduce al reposo en el lecho, la aplicación de dosis más o menos repetidas de cuerpo amarillo, vitamina $\mathrm{E}$ y seclantes de la contración uterina, entre los (najes ol más usado es láudano ingerido o en el clásico enema con cloral. En los nitinos tiempos ha salido al comercio un producto derivado del ácido fosfórice. al betaglimiofosfato de sodio (Elharal), si cual se le atribuye una acción electiva de sedación de la fibra uterina $y$ por consiguionte indicado en las amenazas de aborto y de parto prematuro. La tesis del doctor Quintero Becerra, elahorada el año nasado, versa sobre este tratamiento usando un beta glicerofosfato de sodio, preparado en ampolleta estéril para uso endovenoso, por el dietor Fernando Schoomnewolf. Aun (-nan-

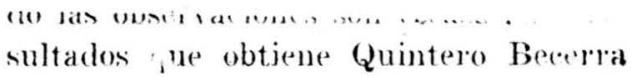
son muy alentadores. Hemos teuido rportmidad de emplear el beta glicerofosfato de sodio en algumas amenazas de parto prematuro, con excelentes resultados; en amenazas de aborto no he. mos tenido la oportmidad de emplearlo.

De toclas maneras, ol tratamiento de Jas amenazas de aborto, sintomático, en m principio, debe orientarse sirmpre bacia la cansa y la determinarión de esta que es difócil y requiere estudios pormenorizados. P'ara no salimos del propósito de estos romentarios, pasamos a lo que hace referencia a la i:onducta ante el aborto inevitable o ante el aborto incompleto. Tenemos a la vista 18 casos de aborto entre ineritables e incompletos, la mayoría incompletos; la conducta que se ha seguido ha sido siempre intervencionista.

Seguros de que el aborto es inevitable o cuando se ha producido parcialmente, no hay duda en que debe intervenirse mediante raspado digital o con cureta; la mayoría de las veces as inrecesario el uso de bujias para dilaiar, porgue en los abortos inevitables y en los incompletos, el enello ya tiene ma dilatación que permite la introduce ón de la cureta grande y roma gue es la más segura. En los rasos a que nos refe. rimos, siempre se ha practicado el raspado ron cureta roma y con la mivor de las curetas de legrados uterinos.

La razón de una conducta interven(ionista por sistema, es la seguridad que se proporciona así (eomo la pronta reposición de las parientes; en efecto: no hay una medicarción que asegure la limpieza de la matriz; si se usan oritivicos para completar el aborto o se expera a que se termine en forma espontinea, 
madie puede asenumar que no queden? restos retenidos y estos restos embrionarios o placentarios, prolongan indefjnidamente las hemorragias, retardas la jnvolución uterina, predisponen a la inferción o la produren de hecho, protongan. deste luégo. el estado de incapariearl de las pareintes, embargan la atemerom ele la fannilia y desesperan al médien. Xo es arenturado pensar que también haty una reperensión endoerina, deste yue no pliede continuar normalmente el ciclo menstrual.

Sin reparar sobre las grandes hemorragias que ponen en peligro la vida de la mujer y sin parar mientes en la infección y sus conseruencias, el solo berho de definir pronto la situación de la pariente en forma rientífica $y$ segmai, instifican tal manera de proceder.

El legrado con cureta, usando la grauif y roma, so de gran seguridad: hay quienes son adictos al raspado digital (•omo menos peligroso y más seguru; sin embargo. no siempre se logra la ni. latación suficiente para la introduceion de dos dedos dentro de la matriz mientras que si se logra la suficiente, maxa la introduceión de la cureta; con el dedo usado para el raspado pueden gion. darse $y$ se quedan grandes islotes 1.. "adilea, los que salen fácilmente eom lit "ureta. Dilatar el vmello hasta podor introducir los dedos no simmpere es far. tible y forzar la dilatarión eon bujiat tiene tántos peligros de muptura norina, y quizás más, que el raspado in trumental. Es natural que quien pras. tica un raspado instrumental debe se? persona capacitada y prudente. P'ara principiantes, desde lnégo, es más segnro el raspado digital deste que que pan Jos dedos.
Para máxima seguridad, después del curetaje se acostumbra dejar un taponamiento con una mecha empapada en penicilina o mojada en aceite estéril y espolvoreada con sulfanilamida en cristales; en ocasiones se deja una mecha embebida en Merthiolate. Este taponaminnto enofiláctico de la infecerón $s$, me eaw die que hayan quedado restos, a fNod del rmetaje, salen al retirar la merha, lo que se hace 12 horas después. Es rarinino que ocurra hemorragia sigruiendo sistemáticamente tal procedimiento: la hemorragia se cohibe con la impieza dis la matriz, pero en el caso contrario el taponamiento lo asegura.

Dosile luegr que a estas pacientes se les atministran sulfanilamidas o penicilina protiláticamente; $y$ no se regnieren mas de tres días de permanenid en lat linica.

Vate lat puar conentar que la casi toialidal di los adsos, se intervinieron iajo anesesia con pentothal, usando una solurión al 2\% y sin pasar de 50 antiglanm en rada intervención. Esta anestesiat es d. rápida aceicon, de rápida climina.ión y de prontat ace ión sobre el Anime perturbato de la pareiente. Por ratain dir lo explesto anters. de que en murehas orasiones no se practira la diJatarion atel evello siempre y cuando gue yllepa la rureta, no es preciso profmdizar la anestesia y esta es ma gajantía más.

Finahnente llamamos la atención sobre la ausencia de infección y sobre el modus operandi para los taponamientos: valva vaginal, pinza de cuello y pinza (3) tapknamiento; no se requiere más instrumental. El taponamiento ron taponalo!n. nunea se ha rejecutado. 